\title{
Efficacy and safety of pitavastatins in patients with acute myocardial infarction: Livalo in Acute Myocardial Infarction Study (LAMIS) II
}

Young Joon Hong ${ }^{1}$, Myung Ho Jeong ${ }^{1}$, Jang Ho Bae ${ }^{2}$, Seok Kyu Oh³ , Seung Woon $\mathrm{Rha}^{4}$, Seung Ho Hur', Sung Yun Lee ${ }^{6}$, Sang Wook Kim7, Kwang Soo Cha ${ }^{8}$, In Ho Chae 9 , Tae Hoon Ahn ${ }^{10}$, and Kee Sik Kim ${ }^{11}$

Department of Cardiology, ${ }^{1}$ Chonnam National University Hospital, Gwangju; ${ }^{2}$ Konyang University Hospital, Daejeon; ${ }^{3}$ Wonkwang University Hospital, Iksan; ${ }^{4}$ Korea University Guro Hospital, Seoul; ${ }^{5}$ Keimyung University Dongsan Medical Center, Daegu; ' Inje University Ilsan Paik Hospital, Goyang; ${ }^{7}$ Chung-Ang University Hospital, Seoul; ${ }^{8}$ Pusan National University Hospital, Busan; ${ }^{9}$ Seoul National University Bundang Hospital, Seongnam; ${ }^{10}$ Gachon University Gil Medical Center, Incheon; ${ }^{11}$ Daegu Catholic University Medical Center, Daegu, Korea

Received: January 25, 2016 Revised : February 12, 2016 Accepted: November 10, 2016

\section{Correspondence to}

Myung Ho Jeong, M.D.

Department of Cardiology,

Chonnam National University

Hospital, 42 Jebong-ro, Dong-gu,

Gwangju 61469, Korea

Tel: +82-62-220-6243

Fax: $+82-62-228-7174$

E-mail: myungho@chollian.net
Background/Aims: We evaluated the efficacy and safety and influence on glucose tolerance by different doses of pitavastatins in acute myocardial infarction (AMI) patients.

Methods: Consecutive 1,101 AMI patients who were enrolled in Livalo in Acute Myocardial Infarction Study (LAMIS)-II were randomly assigned to receive either $2 \mathrm{mg}$ of pitavastatin or $4 \mathrm{mg}$ of pitavastatin orally per day. Primary efficacy endpoint was composite of cardiac death, nonfatal myocardial infarction, target-lesion revascularization, and hospitalization for unstable angina, heart failure or arrhythmic events at 12-month.

Results: There was no significant difference in primary efficacy endpoint between $2 \mathrm{mg}$ and $4 \mathrm{mg}$ groups $(9.07 \%$ vs. $9.13 \%, p=0.976)$. The degree of the reduction of low density lipoprotein cholesterol (LDL-C) was significantly greater in $4 \mathrm{mg}$ group compared to $2 \mathrm{mg}$ group from baseline to follow-up $(-42.05 \pm 32.73 \mathrm{mg} / \mathrm{dL}$ vs. $-34.23 \pm 31.66 \mathrm{mg} / \mathrm{dL}, p=0.002)$. Fasting plasma glucose level was reduced significantly in both groups $(-20.16 \pm 54.49 \mathrm{mg} / \mathrm{dL}$ in $4 \mathrm{mg}$ group and $-24.45 \pm 63.88$ $\mathrm{mg} / \mathrm{dL}$ in $2 \mathrm{mg}$ group, $p<0.001$ and $p<0.001$, respectively) and there was no significant change of glycated hemoglobin in two groups from baseline to follow-up $(-0.13 \% \pm 1.21 \%$ in $4 \mathrm{mg}$ group and $-0.04 \% \pm 1.10 \%$ in $2 \mathrm{mg}$ group, $p=0.256$ and $p=$ 0.671 , respectively).

Conclusions: Although LDL-C was reduced more significantly by using $4 \mathrm{mg}$ of pitavastatin compared to $2 \mathrm{mg}$ of pitavastatin, the event rate was comparable without adverse effects on glucose tolerance in both groups in AMI patients who were enrolled in LAMIS-II.

Keywords: Myocardial infarction; Atherosclerosis; Lipids; Hydroxymethylglutaryl-CoA reductase inhibitors

\section{INTRODUCTION}

Several large, randomized, controlled trials have demonstrated that cholesterol-lowering therapy with 3-hydroxy-3-methylglutaryl coenzyme A reductase inhibi- tors (statins) reduces the risk of death or cardiovascular events [1-7] and intensive lipid-lowering statin regimens provide greater protection against death or major adverse cardiovascular events (MACEs) [8-10] and a slower rate of progression of atherosclerosis than standard reg- 
imens [11-16].

Pitavastatin (LivaloR, Kowa Pharmaceutical Co. Ltd., Kyoto, Japan) is a 3-hydroxy-3-methylglutaryl-coenzyme A reductase inhibitor that significantly reduces the levels of plasma low density lipoprotein cholesterol (LDL-C) and modestly increases levels of high density lipoprotein cholesterol (HDL-C) [17]. Previous Livalo in Acute Myocardial Infarction Study (LAMIS)-I showed favorable clinical outcomes and effective LDL-C lowering, and beneficial effects on the regression and compositional change of coronary plaque after administration of pitavastatin $2 \mathrm{mg} /$ day in acute myocardial infarction (AMI) patients $[18,19]$.

So far, there are very limited data regarding comparison of the efficacy and safety of different doses of pitavastatins in AMI patients. Therefore, the aim of the present study was to evaluate efficacy and safety and influence on glucose tolerance of different doses of pitavastatins in AMI patients who were enrolled in LAMIS-II.

\section{METHODS}

\section{Patient population}

LAMIS is a substudy of Korea Acute Myocardial Infarction Registry (KAMIR). The KAMIR is a Korean prospective multicenter online registry designed to reflect the "real-world" practice in Asian patients presenting with AMI with support from the Korean Circulation Society since November 2005 [20]. Online registry of AMI (at www.kamir.or.kr) has been performed at 55 university or community hospitals that are high-volume centers with facilities for primary percutaneous coronary intervention (PCI) and onsite cardiac surgery. The LAMIS-II investigators were selected from 11 major PCI centers among the 55 KAMIR sites and 1,101 consecutive AMI patients were randomly assigned to receive either $2 \mathrm{mg}$ of pitavastatin or $4 \mathrm{mg}$ of pitavastatin orally daily within 24 hours after PCI between July 2010 and April 2013. The following concomitant medications were limited in the present study to eliminate any possible influence on the efficacy outcomes. (1) Immunosuppressants are prohibited in the present study due to the possibility of rhabdomyolysis as a result of drug interactions between immunosuppressants (e.g., cyclosporine) and statins. If immunosuppressant use is unavoidable, discontinue administration of pitavastatin and continue follow-up. (2) Statins other than pitavastatin are prohibited in the present study due to the possible influence on the evaluation of the efficacy of pitavastatin. (3) The use of lipid-lowering drugs (e.g., bile acid sequestrants or resins), fibric acid derivatives, nicotine acids and derivatives, probucol, etc. were prohibited in the present study because they may influence the evaluation of the efficacy of the study drug. However, additional lipid-lowering drugs can be used only when the investigator decides its use is necessary for the patient. The patients were followed at 30, 90, 180, 270, and 360 days by telephone communication, office visit, or by contacts with primary physicians or referring cardiologists. As part of the protocol, plasma samples were obtained at randomization, 30 and 360 days for the measurements of lipid profiles, fasting plasma glucose (FPG), and glycated hemoglobin (HbA1c). The data were collected electronically at each participating medical centre, transferred to an independent data management organization, and analysed by an independent clinical research organization. The study protocol was approved by the Institutional Review Board of each participating medical centre, and all patients granted their consent to participate in the study.

\section{Sample size calculation}

The present study was designed to compare MACEs between 2 and $4 \mathrm{mg}$ of pitavastatin treatment. Since the aim of the study was to examine the incidence and the odds ratios (ORs) of MACEs between the two dose groups, not to evaluate the statistical differences, sample size was calculated for the estimation of ORs. A meta-analysis of standard and intensive lipid lowering therapies revealed an OR of 0.84 . Therefore, sample size was calculated using the OR of 0.84 as the reference with the precision of the ORs as $25 \%$, considering pitavastatin $2 \mathrm{mg}$ as standard lipid lowering therapy and pitavastatin $4 \mathrm{mg}$ as more intensive lipid lowering therapy. At the significance level of 0.05 , precision of the ORs of 0.25 , and dropout rate of $10 \%$, the minimum number of subjects was calculated as 483 per group.

\section{Efficacy evaluation}

Primary efficacy endpoint (target lesion revascularization [TLR]-MACE) was the incidence of a first MACE, defined as composite of cardiac death, nonfatal myo- 
cardial infarction (MI), TLR, and hospitalization for unstable angina, heart failure, or arrhythmic events at 12-month follow-up. We did not include cardiovascular events at sites outside the stented segment for TLRMACE. Secondary efficacy endpoint was as follows: (1) target vessel revascularization (TVR)-MACE which was defined as the incidence of composite of all-cause death, nonfatal MI, TVR, and hospitalization for unstable angina, heart failure or arrhythmic events at 12-month follow-up; (2) changes of lipid profiles from baseline to 12-month follow-up; and (3) changes of FPG and HbAic from baseline to 12-month follow-up. For TVR-MACE, we included both cardiovascular events in stented segments and those at sites outside the stented segment.

\section{Safety evaluation}

We evaluated the incidence of total adverse events (AEs), serious adverse events (SAEs), and adverse drug reactions (ADRs). AEs for which a causal relationship to pitavastatin could not be ruled out were counted as ADRs. ADRs were reported by the attending physicians. The terminology for ADRs was based on the WHO-ART 092 (WHO Adverse Reaction Terminology).

\section{Statistical analysis}

SAS version 9.2 (SAS Institute Inc., Cary, NC, USA) was used for statistical analysis. The incidence of a first MACE, primary efficacy endpoints, was summarized and the $95 \%$ confidence intervals of OR was calculated. Survival curves of a first MACE and whole cardiovascular events was calculated by Kaplan-Meier and apply log rank test to the comparison between each group. The between-group differences in changes (lipid levels, FPG, and $\mathrm{HbA1c}$ ) from baseline to the 12-month endpoint were assessed by unpaired $t$ test. Paired $t$ tests were used to assess differences between before and after administration of investigational products within groups. All hypotheses were assessed with $5 \%$ of significance level on both sides. While intention-to-treat (ITT) population was defined as the population of patients who had at least one dose of any investigational product administered and had at least one post-baseline measured value of HbA1c along with the baseline level, and ITT population was used for efficacy analysis. Patients who were identified as exclusion criteria deviation during the study and who were not evaluated for efficacy were excluded in the IT'T population. For safety analysis, all randomized patients who had at least one dose of investigational product and had visited after the dose was included. Last observation carried forward (LOCF) was applied for patients who were withdrawn in the middle of the study and LOCF data was used for the results of 1-year endpoint. All data were expressed as mean \pm SD. A $p<0.05$ was considered statistically significant.

\section{RESULTS}

\section{Baseline characteristics}

Fig. 1 shows a flowchart of the patient enrollment and safety and IT'T analyses. The 1,101 patients from 11 facilities in Korea consented to participate in the LAMIS-II. After excluding 55 patients (14 patients, enrolled erroneously; 39 patients, not treated with investigational product; two patients, double randomization), the remaining 1,046 patients were included in the safety analysis. After excluding 68 patients (five patients, violation of inclusion criteria; $\sigma_{3}$ patients, missing of primary efficacy evaluation), the remaining 978 patients were included in the efficacy analysis. The baseline characteristics are summarized in Table 1 . There were no significant differences in age, gender, MI type, and the prevalence of hypertension and diabetes mellitus. Hypercholesterolemia was more prevalent in $4 \mathrm{mg}$ group and current smoking was more prevalent in $2 \mathrm{mg}$ group. There were

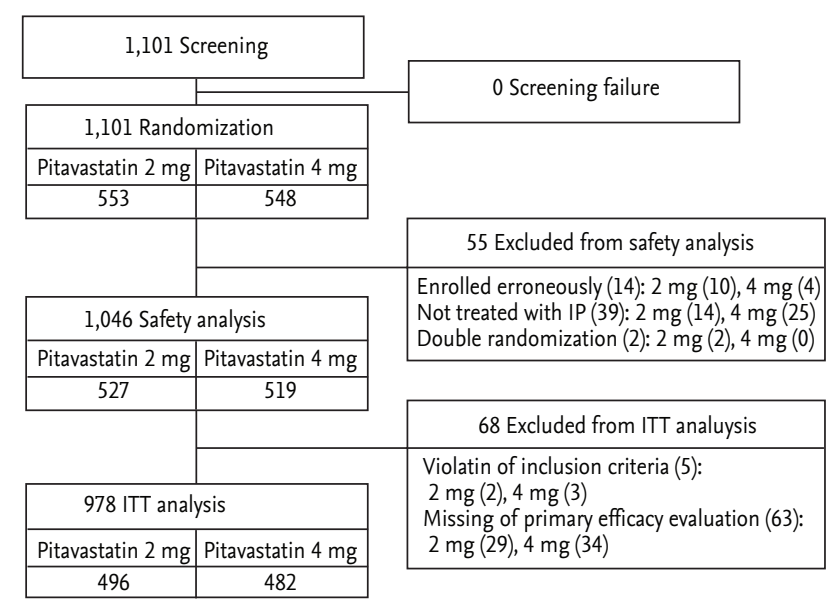

Figure 1. The flow chart of patient enrollment. ITT, intention-to-treat; IP, investigational product. 
Table 1. Baseline characteristics

\begin{tabular}{|c|c|c|c|c|}
\hline Variable & $\operatorname{Total}(\mathrm{n}=978)$ & Pitavastatin $2 \mathrm{mg}(\mathrm{n}=496)$ & Pitavastatin $4 \mathrm{mg}(\mathrm{n}=482)$ & $p$ value \\
\hline Age, yr & $61.06 \pm 11.87$ & $61.41 \pm 11.97$ & $60.70 \pm 11.78$ & 0.345 \\
\hline Sex & & & & 0.852 \\
\hline Male & $731(74.7)$ & $372(75.0)$ & $359(74.5)$ & \\
\hline Female & $247(25 \cdot 3)$ & $124(25.0)$ & $123(25.5)$ & \\
\hline Diagnosis & & & & 0.781 \\
\hline STEMI & $578(59.1)$ & $291(58.7)$ & $287(59 \cdot 5)$ & \\
\hline NSTEMI & $400(40.9)$ & $205(41.3)$ & $195(40.5)$ & \\
\hline Hypertension & $459(46.9)$ & $237(47.8)$ & $222(46.1)$ & 0.404 \\
\hline Diabetes mellitus & $254(26.0)$ & $133(26.8)$ & $121(25.1)$ & 0.542 \\
\hline Hypercholesterolemia & $419(42.8)$ & $205(41.3)$ & $214(44.4)$ & 0.023 \\
\hline Current smoker & $465(47.6)$ & $255(51.4)$ & $210(43.6)$ & 0.002 \\
\hline History of myocardial infarction & $38(3.9)$ & $18(3.6)$ & $20(4.2)$ & 0.929 \\
\hline Thrombolysis & $2(5 \cdot 3)$ & $2(11.1)$ & o & \\
\hline PCI & $30(79.0)$ & $15(83.3)$ & $15(75.0)$ & \\
\hline CABG & $2(5.3)$ & $1(5.6)$ & $1(5.0)$ & \\
\hline Medical therapy & $1(2.6)$ & 0 & $1(5.0)$ & \\
\hline Unknown & $5(13.2)$ & $2(11.1)$ & $3(15.0)$ & \\
\hline History of angina & $584(59 \cdot 7)$ & $296(59.7)$ & $288(59.8)$ & 0.792 \\
\hline Family history of CHD & $74(7.6)$ & $41(8.3)$ & $33(6.9)$ & 0.325 \\
\hline Body mass index, $\mathrm{kg} / \mathrm{m}^{2}$ & $24.25 \pm 3.21$ & $24.26 \pm 3.18$ & $24.25 \pm 3.25$ & 0.934 \\
\hline$<25$ & $597(61.0)$ & $290(58.5)$ & $307(63.7)$ & 0.102 \\
\hline$\geq 25$ & $380(38.9)$ & $205(41.3)$ & $175(36.3)$ & \\
\hline LVEF, \% & $54.58 \pm 10.37$ & $54.58 \pm 10.59$ & $54.59 \pm 10.16$ & 0.982 \\
\hline $\mathrm{CK}, \mathrm{ng} / \mathrm{mL}$ & $572.58 \pm 1,087.64$ & $567.02 \pm 1,048.63$ & $577.60 \pm 1,144.17$ & 0.879 \\
\hline CK-MB, ng/mL & $56.44 \pm 99.58$ & $52.42 \pm 87.43$ & $59.48 \pm 121.38$ & 0.305 \\
\hline Prior history of statin therapy & $98(10.0)$ & $48(9.7)$ & $50(10.4)$ & 0.783 \\
\hline Pitavastatin & $6(6.1)$ & $3(6.3)$ & $3(6.0)$ & \\
\hline Rosuvastatin & $11(11.2)$ & $7(14.6)$ & $4(8.0)$ & \\
\hline Fluvastatin & $1(1.0)$ & $1(2.1)$ & 0 & \\
\hline Atorvastatin & $57(58.2)$ & $26(54.2)$ & $31(62.0)$ & \\
\hline Simvastatin & $8(8.2)$ & $2(4.2)$ & $6(12.0)$ & \\
\hline Unknown & $15(15 \cdot 3)$ & $9(18.8)$ & $6(12.0)$ & \\
\hline Door to PCI time, $\mathrm{hr}$ & & & & 0.559 \\
\hline Within 12 & $680(69.5)$ & $343(69.2)$ & $337(69.9)$ & \\
\hline $12-24$ & $161(16.5)$ & $79(15.9)$ & $82(17.0)$ & \\
\hline $24-48$ & $81(8.3)$ & $47(9.5)$ & $34(7.1)$ & \\
\hline Over 48 & $56(5.7)$ & $27(5.4)$ & $29(6.0)$ & \\
\hline \multicolumn{5}{|l|}{ Discharge medications } \\
\hline Aspirin & $944(95.5)$ & $480(96.8)$ & $464(96.3)$ & 0.664 \\
\hline Clopidogrel & $915(92.5)$ & $459(92.5)$ & $446(92.5)$ & 0.996 \\
\hline Cilostazol & $201(20.6)$ & $100(20.2)$ & $101(21.0)$ & 0.759 \\
\hline Prasugrel & $31(3.2)$ & $16(3.2)$ & $15(3.1)$ & 0.919 \\
\hline$\beta$-Blocker & $756(77 \cdot 3)$ & $383(77.2)$ & $373(77.4)$ & 0.950 \\
\hline Renin-angiotensin system blockers & $731(74.7)$ & $373(75.2)$ & $358(74.3)$ & 0.739 \\
\hline
\end{tabular}

Values are presented as mean \pm SD or number (\%).

STEMI, ST segment myocardial infarction; NSTEMI, non-ST segment myocardial infarction; PCI, percutaneous coronary intervention; CABG, coronary artery bypass graft; CHD, coronary heart disease; LVEF, left ventricular ejection fraction; CK, creatine kinase; $\mathrm{MB}$, myocardial band. 
Table 2. Primary and secondary efficacy endpoint

\begin{tabular}{lccc}
\hline Variable & Pitavastatin $2 \mathrm{mg}(\mathrm{n}=496)$ & Pitavastatin $4 \mathrm{mg}(\mathrm{n}=482)$ & $p$ value \\
\hline Primary efficacy endpoint (TLR-MACE) & $45(9.1)$ & $44(9.1)$ & 0.976 \\
STEMI & $27 / 291(9.3)$ & $26 / 287(9.1)$ & 0.927 \\
NSTEMI & $18 / 205(8.8)$ & $18 / 195(9.2)$ & 0.875 \\
Diabetics & $18 / 133(13.5)$ & $8 / 121(6.6)$ & 0.070 \\
Non-diabetics & $27 / 363(7.4)$ & $36 / 361(10.0)$ & 0.227 \\
Secondary efficacy endpoint (TVR-MACE) & $47(9.5)$ & $47(9.8)$ & 0.884 \\
STEMI & $28 / 291(9.6)$ & $28 / 287(9.8)$ & 0.957 \\
NSTEMI & $19 / 205(9.3)$ & $19 / 195(9.7)$ & 0.871 \\
Diabetics & $19 / 133(14.3)$ & $9 / 121(7.4)$ & 0.082 \\
Non-diabetics & $28 / 363(7.7)$ & $38 / 361(10.5)$ & 0.189 \\
\hline
\end{tabular}

Values are presented as number (\%).

TLR, target lesion revascularization; MACE, major adverse cardiovascular event; STEMI, ST segment myocardial infarction; NSTEMI, non-ST segment myocardial infarction; TVR, target vessel revascularization.

no significant differences in the prior statin therapy and door to PCI time between both groups. There were no significant differences in the discharge medications between both groups. There were no significant differences in the infarct-related artery (although 438 out of 978 patients were investigated for the infarct-related artery) and used stent types between both groups.

\section{Primary efficacy endpoint}

The incidence of primary endpoint is summarized in Table 2. At the time of study termination, 45 first TLRMACE had occurred in 2 mg group, as compared with 44 in $4 \mathrm{mg}$ group ( 2 mg group vs. $4 \mathrm{mg}$ group: OR, $0.993 ; p=$ $0.976)$. In overall population, cardiac mortality rate was $0.6 \%(6 / 978 ; 0.2 \%$ in $2 \mathrm{mg}$ group [1/496] vs. $1.0 \%$ in $4 \mathrm{mg}$ group [5/482], $p=0.119)$. No difference was observed in primary efficacy endpoints in patients with ST segment elevation MI in both 2 and $4 \mathrm{mg}$ groups (OR, 1.027; $p$ $=0.927$ ), and it was also not differently occurred in patients with non-ST segment elevation MI in both 2 and $4 \mathrm{mg}$ groups (OR, $0.947 ; p=0.875$ ). In diabetic subgroups, the incidence of primary efficacy endpoints tended to be lower in $4 \mathrm{mg}$ group compared with $2 \mathrm{mg}$ group (OR, $2.211 ; p=0.070$ ), and no difference was observed in primary efficacy endpoints between 2 and $4 \mathrm{mg}$ groups in non-diabetic subgroups (OR, $0.725 ; p=0.227$ ).

\section{Secondary efficacy endpoint}

The incidence of secondary endpoint in terms of TVR-
MACE is summarized in Table 2. The incidence of TVR-MACE was not different between both groups (OR, $0.969 ; p=0.884)$. In overall population, all-cause mortality rate was $1.1 \%(11 / 978 ; 0.6 \%$ in $2 \mathrm{mg}$ group [3/496] vs. $1.7 \%$ in $4 \mathrm{mg}$ group [8/482], $p=0.118)$. No difference was observed in TVR-MACE in patients with ST segment elevation MI in both 2 and $4 \mathrm{mg}$ groups (OR, 0.985; $p=$ 0.957), and it was also not differently occurred in non-ST segment elevation MI in both 2 and 4 mg groups (OR, $0.947 ; p=0.871)$. The incidence of TVR-MACE tended to be lower in $4 \mathrm{mg}$ group compared with $2 \mathrm{mg}$ group in diabetic subgroups (OR, 2.074; $p=0.082)$, and no difference was observed in TVR-MACE between 2 and $4 \mathrm{mg}$ groups in non-diabetic subgroups (OR, $0.710 ; p=0.189$ ).

Total cholesterol and LDL-C reduced effectively by both 2 and $4 \mathrm{mg}$ of pitavastatin treatment from baseline to 12-month follow-up. The degree of reduction of total cholesterol and LDL-C was significantly greater in 4 $\mathrm{mg}$ group compared with $2 \mathrm{mg}$ group from baseline to 12-month follow-up (Fig. 2A and 2B). At baseline, LDL-C level was $129.06 \pm 35.36$ and $128.46 \pm 38.87 \mathrm{mg} / \mathrm{dL}$ in the pitavastatin $2 \mathrm{mg}$ group and the pitavastatin $4 \mathrm{mg}$ group, respectively. The two groups did not show statistical significance $(p=0.804)$. At 12 months, LDL-C level was 95.21 \pm 27.63 and $86.30 \pm 28.12 \mathrm{mg} / \mathrm{dL}$ in the pitavastatin $2 \mathrm{mg}$ group and the pitavastatin $4 \mathrm{mg}$ group, respectively. The changes in LDL-C at 12 months from baseline were statistically significant in both groups with $-34.23 \pm 31.66$ $\mathrm{mg} / \mathrm{dL}$ in the pitavastatin $2 \mathrm{mg}$ group and $-42.05 \pm 32.73$ 


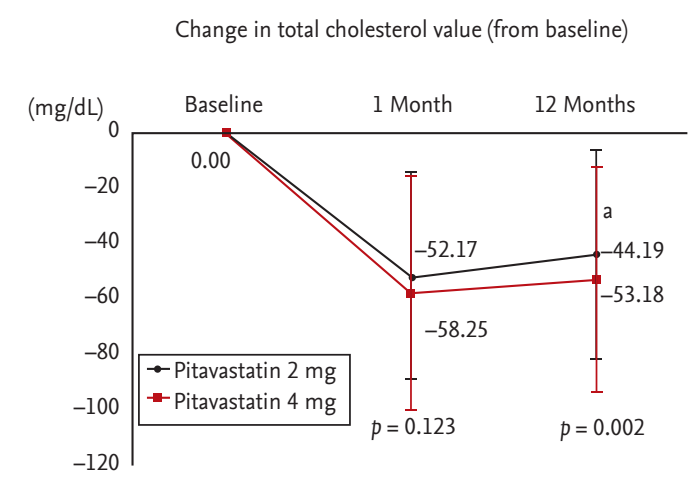

A

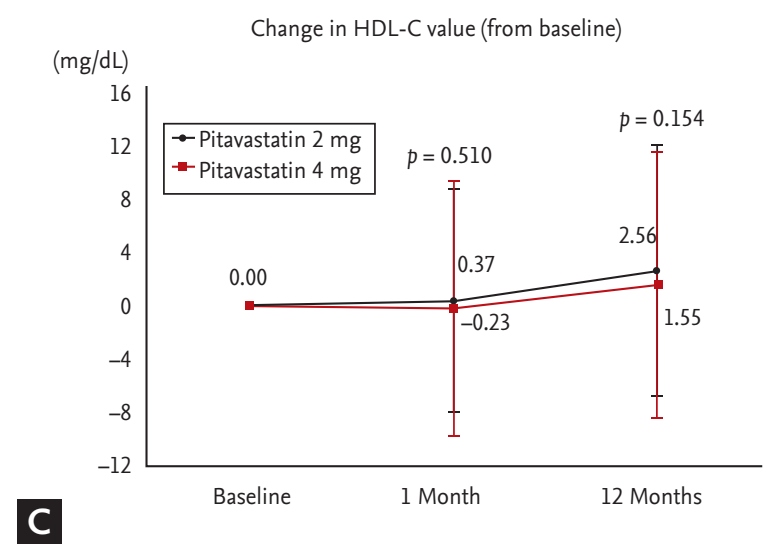

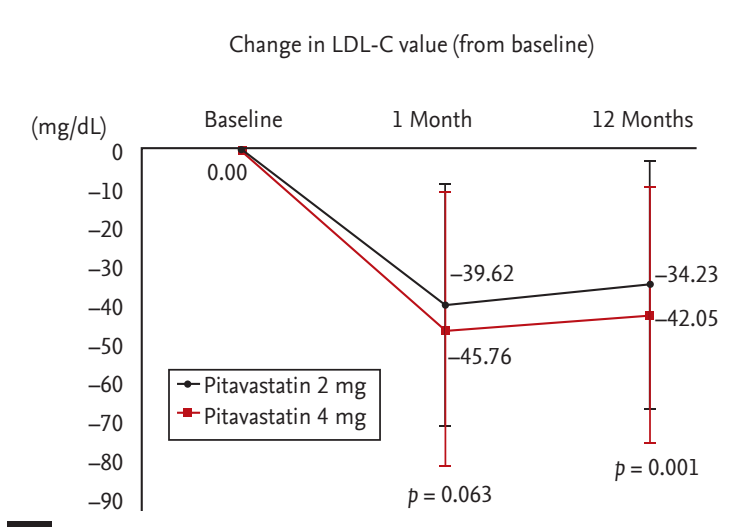

B

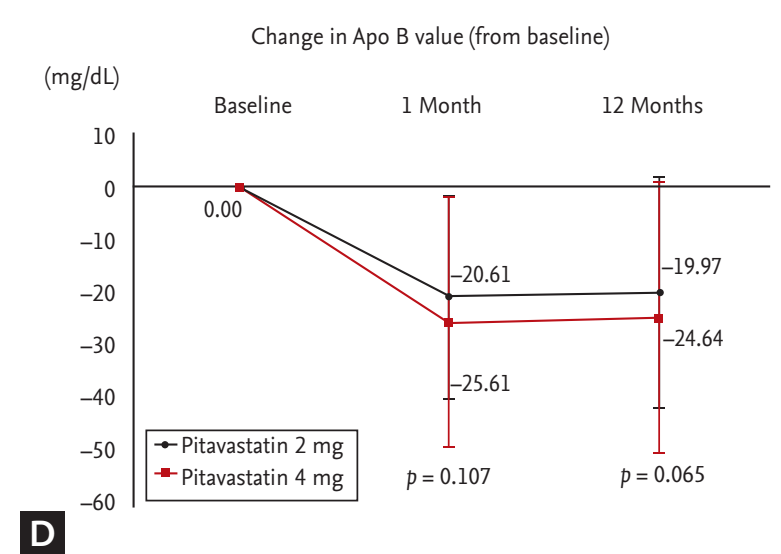

Figure 2. Changes of (A) total cholesterol, (B) low density lipoprotein cholesterol (LDL-C), (C) high density lipoprotein cholesterol (HDL-C), and (D) apolipoprotein B (Apo B) between 2 and $4 \mathrm{mg}$ of pitavastatin groups from baseline to 12 -month follow-up.

$\mathrm{mg} / \mathrm{dL}$ in the pitavastatin $4 \mathrm{mg}$ group $(p<0.001$ and $p$ $<0.001$, respectively). And the change in LDL-C level was significantly greater in the pitavastatin $4 \mathrm{mg}$ group. There was no significant change of triglyceride in both groups from baseline to 12-month follow-up. HDL-C increased effectively by both 2 and $4 \mathrm{mg}$ of pitavastatin treatment from baseline to 12-month follow-up; however, there was no significant difference in change of HDL-C between both groups from baseline to 12-month follow-up (Fig. 2C). Apolipoprotein A1 increased effectively by both 2 and $4 \mathrm{mg}$ of pitavastatin treatment, and apolipoprotein B reduced effectively by both 2 and $4 \mathrm{mg}$ of pitavastatin treatment from baseline to 12-month follow-up; however, there were no significant differences in changes of apolipoprotein A1 and B between both groups from baseline to 12-month follow-up (Fig. 2D).

FPG level was reduced significantly in both 2 and 4 mg group from baseline to 12-month follow-up (Fig. 3A) and $\mathrm{HbAic}$ was not changed significantly in both 2 and $4 \mathrm{mg}$ group. And there were no significant differences in changes of FPG and HbAic between both group from baseline to 12-month follow-up (Fig. 3B). We assessed these parameters separately in diabetic and non-diabetic patients. In diabetic patients, the difference of FPG from baseline to follow-up was $-30.56 \pm 93.67 \mathrm{mg} / \mathrm{dL}$ in pitavastain $2 \mathrm{mg}$ group $(p=0.010)$ and $-20.68 \pm 70.87 \mathrm{mg} / \mathrm{dL}$ in pitavastain $4 \mathrm{mg}$ group $(p=0.044)$. In non-diabetic patients, the difference of FPG from baseline to follow-up was $-20.37 \pm 31.09 \mathrm{mg} / \mathrm{dL}$ in pitavastatin $2 \mathrm{mg}$ group $(p<0.001)$ and $-19.90 \pm 44.65 \mathrm{mg} / \mathrm{dL}$ in pitavastain $4 \mathrm{mg}$ group $(p<$ $0.001)$. In diabetic patients, the difference of $\mathrm{HbAlc}$ from baseline to follow-up was $-0.04 \% \pm 1.31 \%$ in pitavastain 2 mg group $(p=0.829)$ and $-0.30 \% \pm 1.51 \%$ in pitavastain 4 mg group $(p=0.192)$. In non-diabetic patients, the difference of $\mathrm{HbA1c}$ from baseline to follow-up was $-0.05 \% \pm$ $0.89 \%$ in pitavastain $2 \mathrm{mg}$ group $(p=0.675)$ and $-0.02 \% \pm$ 

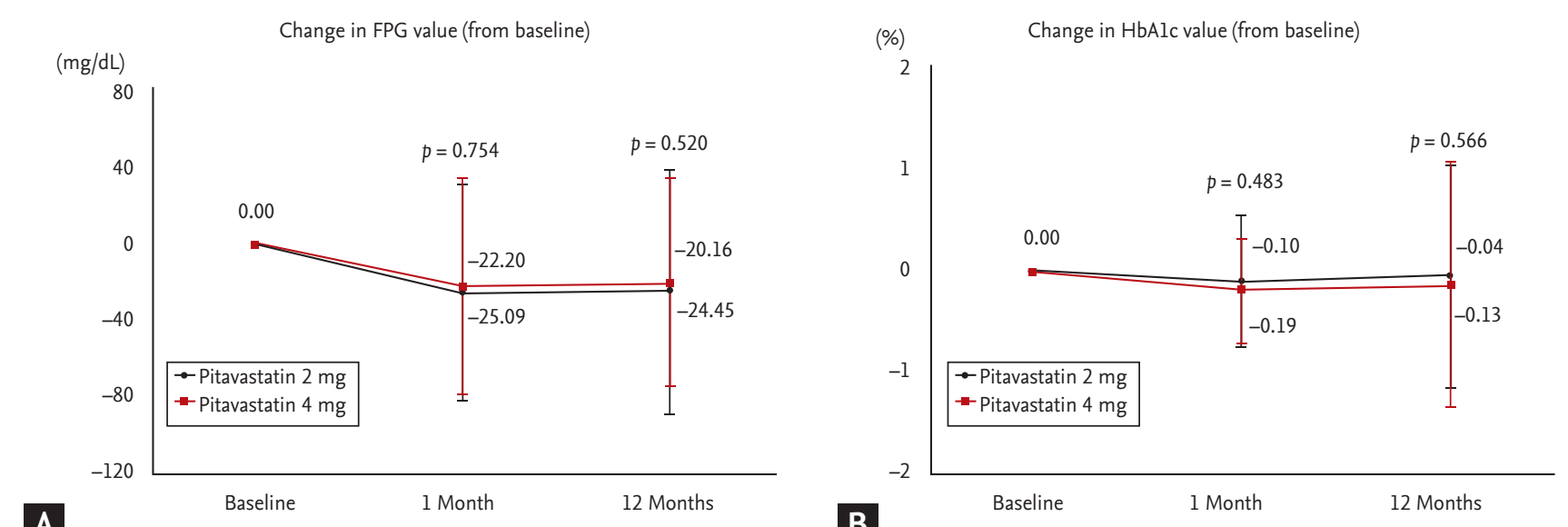

\section{a}

Figure 3. Changes of (A) fasting plasma glucose (FPG) and (B) glycated hemoglobin (HbA1c) between 2 and 4 mg of pitavastatin groups from baseline to 12-month follow-up.

$0.96 \%$ in pitavastain $4 \mathrm{mg}$ group $(p=0.871)$

\section{Kaplan-Meier estimates}

On the basis of Kaplan-Meier estimates, there were no significant differences of occurrence of TLR-MACE, TVR-MACE, cardiac death, and nonfatal MI between both groups (Fig. 4).

\section{Safety endpoint}

Among 1,101 subjects randomized in the study, 1,046 (95.0\%) were included in the safety set except for those who were with double randomization, enrolled erroneously or not treated with pitavastatin ( $n=527$ vs. 519 for pitavastatin $2 \mathrm{mg}$ and pitavastatin $4 \mathrm{mg}$ groups, respectively).

In the present study, 114/527 subjects (21.6\%, 166 events) in the pitavastatin $2 \mathrm{mg}$ group and $115 / 519(22.2 \%$, 147 events) in the pitavastatin $4 \mathrm{mg}$ group experienced at least one AEs; there was no statistically significant difference in the incidence of the AEs between two groups $(p=0.837)$. There was also no statistically significant difference in the incidence of the ADRs between the pitavastatin $2 \mathrm{mg}$ group (10/527 [1.9\%], 15 reactions) and the pitavastatin $4 \mathrm{mg}$ group $(8 / 519$ [1.5\%], 8 reactions; $p$ $=0.658$ ).

The SAEs were reported in 76/527 subjects (14.4\%, 99 events) in the pitavastatin $2 \mathrm{mg}$ group and $77 / 519$ (14.8\%, 90 events) in the pitavastatin $4 \mathrm{mg}$ group; similarly, there was no statistically significant difference in the incidence of the SAEs between two groups $(p=0.849)$.
Among the SAEs, three events were related to the study drug at the discretion of the investigator, including 'urticaria' in pitavastatin $2 \mathrm{mg}$ group (subject no. So4-121) and 'myocardial infarction' and 'malignant lymphoma' in pitavastatin $4 \mathrm{mg}$ group (subject no. So8-059 and S11022, respectively).

The incidence of ADR by system organ class (SOC) in safety population is summarized in Table 3. When all ADRs were standardized by SOC and preferred term using WHO-ART 092, the most common SOCs were 'myoendopericardial \& valve disorders' (seven events) followed by 'musculoskeletal system disorders' (five events), 'body as a whole: general disorders' (three events), and 'liver and biliary system disorders' (three events).

\section{DISCUSSION}

The LAMIS-II is the first investigation conducted in Korea for the evaluation of the incidence of events that occur during treatment and effects on lipid profiles and glucose tolerance with different doses of pitavastatins in AMI patients. The present LAMIS-II demonstrated that (1) the incidences of cardiac mortality and all-cause mortality were very low in both 2 and 4 mg of pitavastatin groups, (2) the incidences of TLR-MACE and TVRMACE were not significantly different between 2 and 4 mg of pitavastatin groups, and (3) both 2 and 4 mg of pitavastatin treatment reduced LDL-C and FPG effectively 
A
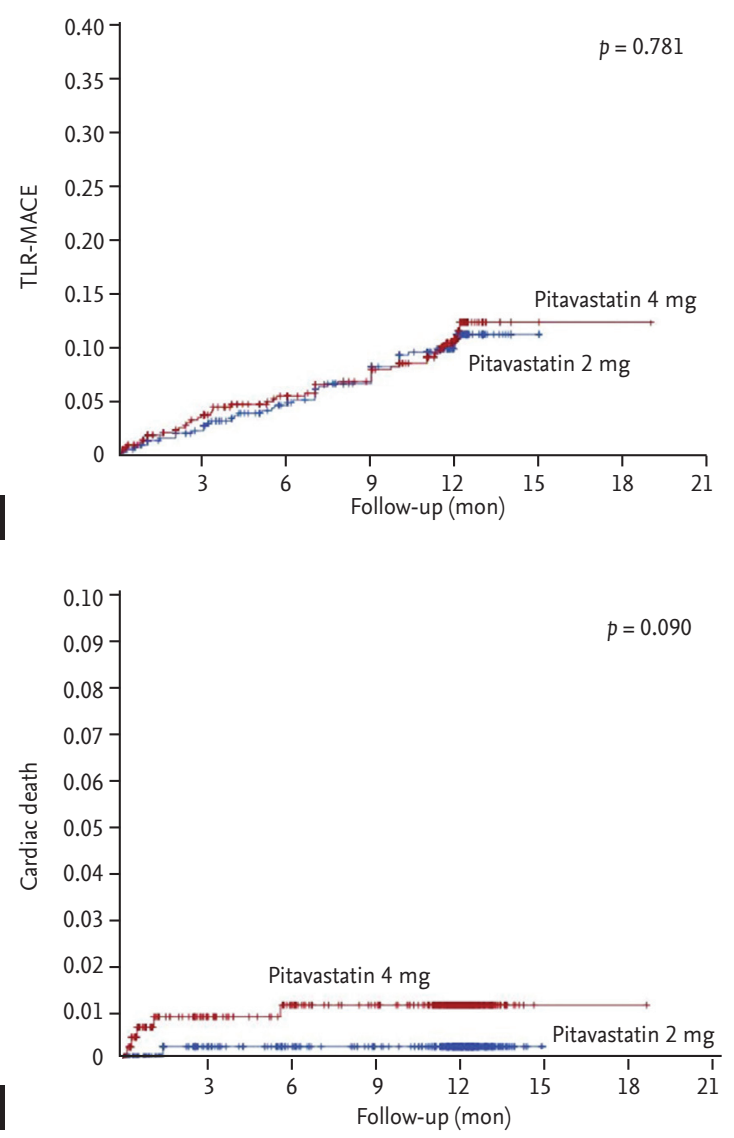
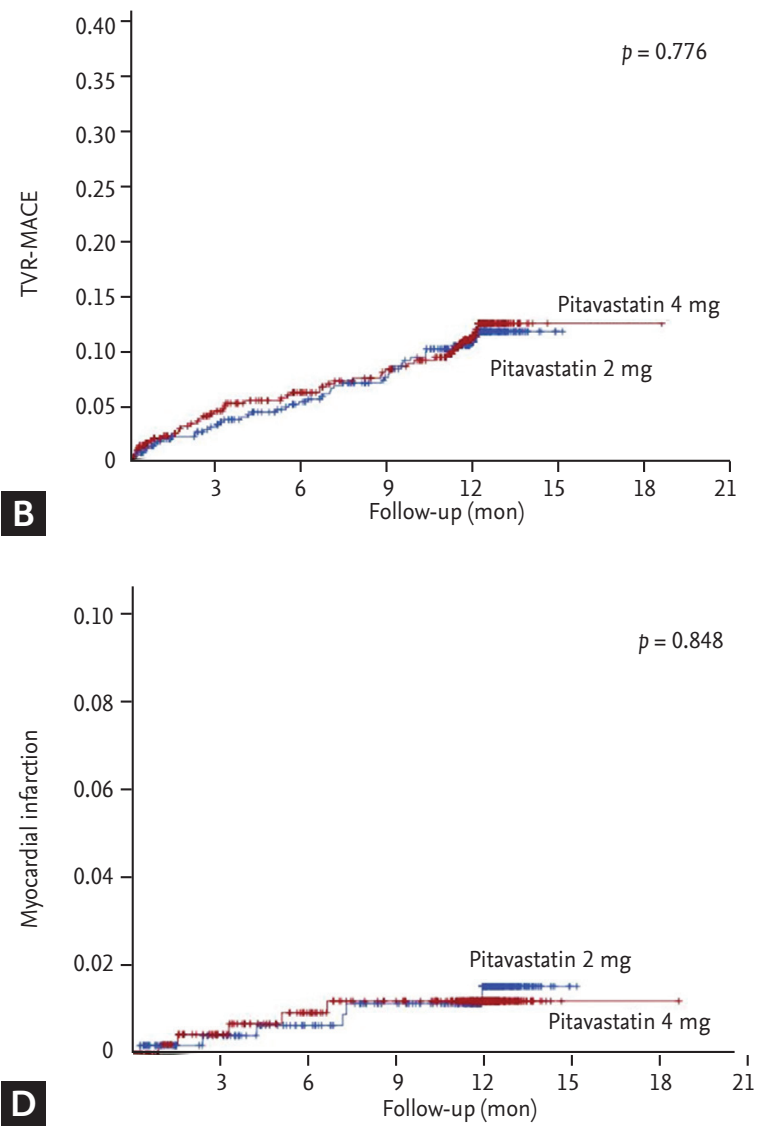

Figure 4. Kaplan-Meier estimates of the incidences of (A) target lesion revascularization-major adverse cardiovascular event (TLR-MACE), (B) target vessel revascularization (TVR)-MACE, (C) cardiac mortality, and (D) myocardial infarction between 2 and $4 \mathrm{mg}$ of pitavastatin groups at 12-month follow-up.

from baseline to 12-month follow-up.

LAMIS-I demonstrated that administration of pitavastatin $2 \mathrm{mg} /$ day in patients with AMI showed 70.5\% LDL-C target attainment with good tolerance and was associated with favorable clinical outcomes up to 12 months [18]. Another study from LAMIS-I demonstrated that reduction in high-sensitivity C-reactive protein levels in AMI patients plays an important role in the beneficial effects of pitavastatin on the regression and compositional change of coronary plaque [19]. In the present LAMIS-II, we evaluated efficacy and safety and effects on glucose tolerance by 2 and $4 \mathrm{mg}$ of pitavastatin treatment in AMI patients.

In previous LAMIS-I [18], all-cause deaths occurred in $3.5 \%$ of patients including $2.1 \%$ of cardiac deaths after administration of pitavastatin $2 \mathrm{mg} /$ day in patients with AMI. In the present LAMIS-II, we observed very low rate of cardiac mortality and all-cause mortality after the use of both 2 and $4 \mathrm{mg}$ of pitavastatin in AMI patients. Because four deaths occurred after study discontinuation due to SAEs, we did not include these deaths into occurrence of events. In the present study, more effective lowering of LDL-C was observed in $4 \mathrm{mg}$ group compared with $2 \mathrm{mg}$ group from baseline to 1-year follow-up; however, there was no difference in TLR-MACE and TVRMACE between 2 and $4 \mathrm{mg}$ of pitavastatin groups in AMI patients. The reason for these no differences in event rates between 2 and $4 \mathrm{mg}$ of pitavastatin treatment is not clear. However, we used second generation drug-eluting stents in more than $90 \%$ of the enrolled patients and many medications, including aspirin, platelet adenosine diphosphate receptor antagonist, renin-angiotensin system blocker and so on, which showed beneficial effects in AMI patients were used in most of the enrolled patients.

JUPITER (Justification for the Use of Statins in Prima- 
Table 3. Adverse drug reaction by system organ class (safety population)

\begin{tabular}{|c|c|c|c|}
\hline Variable & Pitavastatin $2 \mathrm{mg}(\mathrm{n}=527)$ & Pitavastatin $4 \mathrm{mg}(\mathrm{n}=519)$ & Total $(n=1,046)$ \\
\hline Myoendopericardial \& valve disorders & $6(5 \cdot 3)$ & $1(0.9)$ & $7(3.0)$ \\
\hline Myocardial infarction & o & $1(0.9)$ & $1(0.4)$ \\
\hline AST increased & $3(2.6)$ & o & $3(1.3)$ \\
\hline ALT increased & $3(2.6)$ & o & $3(1.3)$ \\
\hline Body as a whole: general disorders & $1(0.9)$ & $2(1.7)$ & $3(1.3)$ \\
\hline Chest pain & $1(0.9)$ & o & $1(0.4)$ \\
\hline Asthenia & o & $1(0.9)$ & $1(0.4)$ \\
\hline Fatigue & ० & $1(0.9)$ & $1(0.4)$ \\
\hline Musculoskeletal system disorders & $3(2.6)$ & $2(1.7)$ & $5(2.2)$ \\
\hline Myalgia & $3(2.6)$ & $2(1.7)$ & $5(2.2)$ \\
\hline Skin and appendages disorders & $2(1.8)$ & o & $2(0.9)$ \\
\hline Pruritus & $1(0.9)$ & o & $1(0.4)$ \\
\hline Urticaria & $1(0.9)$ & o & $1(0.4)$ \\
\hline Liver and biliary system disorders & $2(1.8)$ & $1(0.9)$ & $3(1.3)$ \\
\hline Hepatic enzymes increased & $2(1.8)$ & $1(0.9)$ & $3(1.3)$ \\
\hline Neoplasm & o & $1(0.9)$ & $1(0.4)$ \\
\hline Lymphoma malignant & o & $1(0.9)$ & $1(0.4)$ \\
\hline Metabolic and nutritional disorders & $1(0.9)$ & $1(0.9)$ & $2(0.9)$ \\
\hline Diabetes mellitus & & $1(0.9)$ & $1(0.4)$ \\
\hline Creatine phosphokinase increased & $1(0.9)$ & o & $1(0.4)$ \\
\hline Total & $15(2.9)$ & $8(1.5)$ & $23(2.2)$ \\
\hline
\end{tabular}

Values are presented as number (\%).

AST, aspartate aminotransferase; ALT, alanine aminotransferase.

ry Prevention) trial [21] reported a significant increase in the rate of physician-reported diabetes mellitus with rosuvastatin, as well as significant increase in the median value of $\mathrm{HbArc}$. Increases in glucose and $\mathrm{HbAlc}$ levels, the incidence of newly diagnosed diabetes mellitus, and worsening glycemic control have been reported in previous trials of atorvastatin $[22,23]$. In the PATROL (Randomized Head-to-Head Comparison of Pitavastatin, Atorvastatin, and Rosuvastatin for Safety and Efficacy [Quantity and Quality of LDL]) trial [24], HbAlc was significantly increased in the atorvastatin and rosuvastatin groups, but there was no change in the pitavastatin group. In the present study, $4 \mathrm{mg}$ of pitavastatin reduced FPG effectively and HbAic levels were reduced a little. The exact mechanisms about the beneficial effects of pitavastatin on glucose tolerance over other statins such as rosuvastatin and atorvastatin are not well known, but several postulated mechanisms are suggested. In vitro study demonstrated that atorvastatin suppressed glu- cose transporter type 4 (GLUT-4) expression in $3 \mathrm{~T}_{3}-\mathrm{L} 1$ adipose cells; therefore, atorvastatin accelerated glucose intolerance as a result of insulin resistance in the peripheral tissue [25]. In contrast, pitavastatin did not impair the differentiation and maturation of $3 \mathrm{~T}_{3}-\mathrm{L} 1$ preadipocytes, and it did not suppress GLUT-4 expression when used at clinical concentrations $[26,27]$. In the present study, HDL-C increased effectively by both 2 and 4 $\mathrm{mg}$ of pitavastatin treatment from baseline to 12-month follow-up. Both HDL-C and apolipoprotein Aı increase glucose uptake in primary human skeletal muscle cell cultures established from patients with type 2 diabetes mellitus [28].

There are several limitations to be mentioned. First, the present study was not adequately powered to detect changes in the risk of death from any cause. The rates of death in both groups were very low as compared with those in previous secondary-prevention trials of statins, accounting for only about one-thirds of all deaths. 
Therefore, no significant differences were observed between the two pitavastatin groups in the incidences of cardiac deaths and all-cause deaths. We cannot rule out the possibility that the incidence of events was underestimated in the present study. However, the objective of the present study was to estimate the OR of MACE in the two groups, the pitavastatin $2 \mathrm{mg}$ group and the pitavastatin $4 \mathrm{mg}$ group, not to compare the incidence rates of MACE. So, when sample size was calculated, the power was not considered. Since comparison of incidence rates of MACE between the two groups was not the objective of the study, we believed power calculation was not necessary. However, we calculated the difference in the incidence of MACE between the two groups as well as their $p$ values in addition. Also, the design of the previous study, LAMIS-I, involved dose escalation from pitavastatin 2 to $4 \mathrm{mg}$, which made it difficult to calculate the incidence of MACE for 2 and $4 \mathrm{mg}$, respectively. This means that sample size estimation could not be done for this study based on LAMIS-I. This is the reason why the objective of the present study was to estimate the ORs of MACE in the two groups. Second, in the present study, pitavastatin treatment showed effective sugar marker reduction in AMI patients at 12-month follow-up; however, long-term follow-up may be needed to evaluate the effects of pitavastatin on development of new-onset diabetes mellitus.

In conclusion, although LDL-C was reduced more significantly by using $4 \mathrm{mg}$ of pitavastatin compared to 2 $\mathrm{mg}$ of pitavastatin, the incidence of MACE was not different with very low rate of mortality and effective lowering of FPG level in both groups in AMI patients who were enrolled in LAMIS-II.

\section{KEY MESSAGE}

1. The Livalo in Acute Myocardial Infarction Study (LAMIS)-II is the first study which was conducted for the evaluation of the incidence of cardiovascular events and effects on lipid profiles and glucose tolerance with two doses of pitavastatins in Korean acute myocardial infarction patients.

2. The LAMIS-II demonstrated very low incidences of cardiac mortality and all-cause mortality by using both 2 and $4 \mathrm{mg}$ of pitavastatins.
3. The LAMIS-II demonstrated that the incidences of major adverse cardiovascular event were not significantly different between 2 and $4 \mathrm{mg}$ of pitavastatin groups.

4. The LAMIS-II demonstrated that both 2 and 4 mg of pitavastatin treatment reduced low density lipoprotein cholesterol effectively without adverse effects on glucose tolerance from baseline to 12-month follow-up.

\section{Conflict of interest}

No potential conflict of interest relevant to this article was reported.

\section{Acknowledgments}

This study was supported by a grant of the Korean Health Technology R\&D Project, Ministry of Health and Welfare, Republic of Korea (HI13C1527), and a grant of the Korean Health Technology R\&D Project (HI14C2069), and a grant of the National Research Foundation of Korea Grant funded by the Korean Government (2011ooo8875), and a grant of the Korean Health Technology R\&D Project, Ministry of Health and Welfare, Republic of Korea (HI13 $\mathrm{Co}_{16}$ ), and a grant of the Bio \& Medical Technology Development Program of the National Research Foundation (NRF) funded by the Korean government (MEST) (2012M3A9C6049744), and a grant of Chonnam National University Hospital Research Institute of Clinical Medicine (CRI 11080-21), Republic of Korea.

\section{REFERENCES}

1. The Scandinavian Simvastatin Survival Study (4S). Randomised trial of cholesterol lowering in 4444 patients with coronary heart disease. Lancet 1994;344:1383-1389.

2. Sacks FM, Pfeffer MA, Moye LA, et al. The effect of pravastatin on coronary events after myocardial infarction in patients with average cholesterol levels: Cholesterol and Recurrent Events Trial investigators. N Engl J Med 1996;335:1001-1009.

3. Long-Term Intervention with Pravastatin in Ischaemic Disease (LIPID) Study Group. Prevention of cardiovas- 
cular events and death with pravastatin in patients with coronary heart disease and a broad range of initial cholesterol levels. N Engl J Med 1998;339:1349-1357.

4. Heart Protection Study Collaborative Group. MRC/BHF Heart Protection Study of cholesterol lowering with simvastatin in 20,536 high-risk individuals: a randomised placebo-controlled trial. Lancet 2002;360:7-22.

5. Shepherd J, Cobbe SM, Ford I, et al. Prevention of coronary heart disease with pravastatin in men with hypercholesterolemia: West of Scotland Coronary Prevention Study Group. N Engl J Med 1995;333:1301-1307.

6. Downs JR, Clearfield M, Weis S, et al. Primary prevention of acute coronary events with lovastatin in men and women with average cholesterol levels: results of AFCAPS/TexCAPS. Air Force/Texas Coronary Atherosclerosis Prevention Study. JAMA 1998;279:1615-1622.

7. Shepherd J, Blauw GJ, Murphy MB, et al. Pravastatin in elderly individuals at risk of vascular disease (PROSPER): a randomised controlled trial. Lancet 2002;360:1623-1630.

8. Cannon $\mathrm{CP}$, Braunwald $\mathrm{E}, \mathrm{McCabe} \mathrm{CH}$, et al. Intensive versus moderate lipid lowering with statins after acute coronary syndromes. N Engl J Med 2004;350:1495-1504.

9. Ridker PM, Cannon CP, Morrow D, et al. C-reactive protein levels and outcomes after statin therapy. N Engl J Med 2005;352:20-28.

10. LaRosa JC, Grundy SM, Waters DD, et al. Intensive lipid lowering with atorvastatin in patients with stable coronary disease. N Engl J Med 2005;352:1425-1435.

11. Nissen SE, Tuzcu EM, Schoenhagen P, et al. Effect of intensive compared with moderate lipid-lowering therapy on progression of coronary atherosclerosis: a randomized controlled trial. JAMA 2004;291:1071-1080.

12. Nissen SE, Tuzcu EM, Schoenhagen P, et al. Statin therapy, LDL cholesterol, C-reactive protein, and coronary artery disease. N Engl J Med 2005;352:29-38.

13. Nissen SE, Nicholls SJ, Sipahi I, et al. Effect of very high-intensity statin therapy on regression of coronary atherosclerosis: the ASTEROID trial. JAMA 2006;295:15561565 .

14. Lee SW, Hau WK, Kong SL, et al. Virtual histology findings and effects of varying doses of atorvastatin on coronary plaque volume and composition in statin-naive patients: the VENUS study. Circ J 2012;76:2662-2672.

15. Natsuaki M, Furukawa $Y$, Morimoto T, et al. Intensity of statin therapy, achieved low-density lipoprotein cholesterol levels and cardiovascular outcomes in Japanese pa- tients after coronary revascularization: perspectives from the CREDO-Kyoto registry cohort-2. Circ J 2012;76:13691379 .

16. Okada K, Ueda Y, Takayama T, et al. Influence of achieved low-density lipoprotein cholesterol level with atorvastatin therapy on stabilization of coronary plaques: sub-analysis of the TWINS study. Circ J 2012;76:1197-1202.

17. Noji Y, Higashikata T, Inazu A, et al. Long-term treatment with pitavastatin (NK-104), a new HMG-CoA reductase inhibitor, of patients with heterozygous familial hypercholesterolemia. Atherosclerosis 2002;163:157-164.

18. Suh SY, Rha SW, Ahn TH, et al. Long-term safety and efficacy of Pitavastatin in patients with acute myocardial infarction (from the Livalo Acute Myocardial Infarction Study [LAMIS]). Am J Cardiol 2011;108:1530-1535.

19. Hong YJ, Jeong MH, Ahn Y, et al. Effect of pitavastatin treatment on changes of plaque volume and composition according to the reduction of high-sensitivity C-reactive protein levels. J Cardiol 2012;60:277-282.

20. Chen KY, Rha SW, Li YJ, et al. Triple versus dual antiplatelet therapy in patients with acute ST-segment elevation myocardial infarction undergoing primary percutaneous coronary intervention. Circulation 2009;119:3207-3214.

21. Ridker PM, Danielson E, Fonseca FA, et al. Rosuvastatin to prevent vascular events in men and women with elevated C-reactive protein. N Engl J Med 2008;359:21952207.

22. Sasaki J, Iwashita M, Kono S. Statins: beneficial or adverse for glucose metabolism. J Atheroscler Thromb 2006;13:123-129.

23. Yamakawa T, Takano T, Tanaka S, Kadonosono K, Terauchi Y. Influence of pitavastatin on glucose tolerance in patients with type 2 diabetes mellitus. J Atheroscler Thromb 2008;15:269-275.

24. Saku K, Zhang B, Noda K; PATROL Trial Investigators. Randomized head-to-head comparison of pitavastatin, atorvastatin, and rosuvastatin for safety and efficacy (quantity and quality of LDL): the PATROL trial. Circ J 2011;75:1493-1505.

25. Nakata M, Nagasaka S, Kusaka I, Matsuoka H, Ishibashi $\mathrm{S}$, Yada T. Effects of statins on the adipocyte maturation and expression of glucose transporter 4 (SLC2A4): implications in glycaemic control. Diabetologia 2006;49:18811892.

26. Ishihara $Y$, Ohmori K, Mizukawa M, Hasan AU, Noma T, Kohno M. Beneficial direct adipotropic actions of pi- 
tavastatin in vitro and their manifestations in obese mice. Atherosclerosis 2010;212:131-138.

27. Mita T, Nakayama S, Abe H, et al. Comparison of effects of pitavastatin and atorvastatin on glucose metabolism in type 2 diabetic patients with hypercholesterolemia. J
Diabetes Investig 2013;4:297-303.

28. Drew BG, Duffy SJ, Formosa MF, et al. High-density lipoprotein modulates glucose metabolism in patients with type 2 diabetes mellitus. Circulation 2009;119:2103-2111. 